\section{$011 \quad$ OT PEAK DISPERSION CORRELATED WITH LV SIZE AND MASS IN PATIENTS WITH CHRONIC HEART FAILURE, BUT NOT TO THE AMOUNT OF SCAR PRESENT}

O Khaleva, ${ }^{1}$ N Sherwi, ${ }^{1}$ E Lukaschuk, ${ }^{1}$ M Borovickova, ${ }^{1}$ Z H Khan, ${ }^{1}$ V Lee, ${ }^{2}$ A Joshi, ${ }^{1}$ H Loh, ${ }^{1}$ C Bourantas, ${ }^{1}$ A L Clark, ${ }^{1}$ J G F Cleland, ${ }^{1}$ K Wong ${ }^{1}$ Hull York Medical School, University of Hull, ${ }^{2}$ Hull York Medical School, University of York

doi:10.1136/heartjnl-2013-304019.11

Introduction In middle aged men with a normal OT interval on a resting 12-lead ECG, increased OT peak dispersion is an independent risk factor for sudden cardiac death. OT peak prolongation is associated with left ventricular (LV) hypertrophy in hypertensive subjects, stroke survivors, and patients with suspected coronary artery disease. However, young footballers with LVH characterised by myocyte hypertrophy (with no fibrosis) do not have QT peak prolongation. We wondered if increased left ventricular mass and scar burden were associated with QT peak dispersion in patients with heart failure (HF).

Methods 121 consecutive patients with chronic heart failure in sinus rhythm were studied. LV mass was determined on cardiac MRI. Scar mass was assessed using late gadolinium enhancement. The ECGs were analysed by a single observer who was blind to the results of the cardiac MRI and to clinical findings. OT peak (QTp) was measured from onset of the $\mathrm{Q}$-wave to the peak of $\mathrm{T}$-wave in every ECG lead of the 12-lead ECG, and heart rate corrected (OTpc) using Bazett's formula. OTpc dispersion was defined as QTpc Max-OTpc Min. Results are presented as mean $\pm \mathrm{SD}$, unless stated otherwise.

Results 121 patients (17 female) were aged $66 \pm 10.9$ years. LV ejection fraction was (EF) $36 \pm 11 \%$, end-diastolic volume (EDV) $239 \pm 72 \mathrm{ml}$, end-systolic volume (ESV) $159 \pm 67 \mathrm{ml}$, LV mass $146 \pm 30.9 \mathrm{~g}$, scar mass was $27 \pm 10 \mathrm{~g}$ and formed $19 \pm 7 \%$ of the LV mass. The OTpc dispersion was $76 \pm 40 \mathrm{~ms}$.

QTpc dispersion correlated with total LV mass $(r=0.3, p=0.01)$ and size (EDV $r=0.4, p=0.002 ; E S V ~ 0.4, p=0.001$ ). There was no correlation between OTpc dispersion and scar size, either expressed in absolute units or as a percentage of total LV mass.

Conclusions QTpc dispersion correlated with the total LV mass and LV size. However, there was no correlation between OTpc dispersion and scar size as determined by cardiac MR with late gadolinium enhancement imaging. 